\title{
Report of the IAU Working Group on 'Nonrigid-Earth Nutation Theory'
}

\author{
Véronique Dehant \\ Royal Observatory of Belgium, 3 avenue Circulaire, B1180 Brussels, \\ Belgium
}

\begin{abstract}
.
The last precession-nutation model adopted by the IAU (International Astronomical Union) in 1980 is the nutation series built on Wahr's Earth transfer function for the nutations of an oceanless elastic Earth (Wahr, 1979, 1981), and on Kinoshita's rigid-Earth precession-nutation series (Kinoshita, 1977; Kinoshita et al., 1979). The resulting precession and nonrigid-Earth nutation series have been used since that time and have been compared with observations. This comparison, which has been done by different teams all-over the world, shows that the theoretical series must be improved to meet observational precision. A Working Group (WG) was set up to examine the possibility of adopting a new nonrigid-Earth nutation series and to study the existing possibilities. On the one hand, the rigid-Earth nutation series have been improved (three new series) and the mutual differences have been shown to be less than a few hundreds of microarcseconds. On the other hand, new Earth transfer functions have been derived based on additional physical considerations within the Earth. The problem with these transfer functions however is that there is no reliable, independent information about the geophysical parameters needed to improve a theoretical model. Instead, the discrepancies with the nutation observations themselves are used to infer those parameters. Recent fits of geophysical parameters to the observed nutations have provided a series that is suitable for practical use, and is also a source of important information on the physics of the Earth's interior. This paper reviews the recent work of the WG and establishes the reasons and criteria for the choice of the new model 'IAU 2000' which is proposed for adoption at the next IAU General Assembly.
\end{abstract}

\section{Introduction}

The last nutation theory that has been adopted is the theory of Wahr (1981) for an ellipsoidal deformable and uniformly rotating Earth convolved with Kinoshita's (1977) rigid-Earth nutation series. This theory was adopted in 1980 by the IAU. The advance of VLBI (Very Long Baseline Interferometry) techniques to observe the Earth's orientation parameters including the nutations, has led to the designation, in 1994 (IAU General Assembly) of a WG entitled 'Nonrigid Earth Nutation Theory' to examine the possibilities for more precise nonrigid- 
Earth nutation theories. This WG is a joint IAU-IUGG WG. The "terms of reference" are then to try to obtain a new nonrigid-Earth nutation theory which could be used to give the Earth's orientation in space to users who want high accuracy, especially for geophysical and geodetic studies. The WG has been very active during these years. In particular, several new theories have appeared very recently. In this paper I will briefly review the conclusions of the WG as presented in a paper (Dehant et al., 1999) and present the recent theories that have been developed. This will lead to the description of the resolution adopted during IAU Colloquium 180.

The nutation of a nonrigid Earth is due to the lunisolar and planetary attraction and to the oceanic and the atmospheric forcing. The WG has been working at six different levels:

- Level 1 concerns the input models (giving profiles of the Earth's density and rheological properties) for the calculation of the Earth's transfer functions of Level 2.

- Level 2 concerns the integration inside the Earth in order to obtain the Earth's transfer functions for the nutations at different frequencies.

- Level 3 concerns the rigid-Earth nutations.

- Level 4 examines the convolution (products in the frequency domain) between the Earth's nutation transfer functions obtained in Level 2, and the rigid-Earth nutation obtained in Level 3. This is for an Earth without oceans and atmosphere.

- Level 5 concerns the effects of the atmosphere and the oceans on the precession, obliquity rate, and nutations.

- Level 6 concerns the comparison with the VLBI observations, of the theoretical results obtained in Level $4+$ the corrections obtained in Level 5. levels.

The main results of the work of this WG are described here for the different

\section{Level 1: seismic model}

In this level we have examined the different seismic models giving rise to spherical Earth rheological profiles, including the density profile. In particular, we have shown that the present-day seismic models for a spherical Earth that are used to obtain the elliptical Earth results (using Clairaut's equation for hydrostatic equilibrium), are not sufficient to explain the observed dynamical flattening, as well as the inferred Core-Mantle Boundary (CMB) flattening or Inner Core Boundary (ICB) flattening. It is necessary to consider that the Earth is not in hydrostatic equilibrium at the initial state. 


\section{Level 2: transfer function}

The transfer functions are presently of three kinds:

(1) The "empirical" formula based on a simple resonance where the parameters are fit to VLBI data. This was the case for the 1996 IERS model and is the case for two new upgraded computations from Herring (2000) and Shirai and Fukushima (2000a). By construction, these models are very close to the observations, but the parameters cannot be interpreted in terms of physics of the Earth's interior.

(2) The so-called "B-ratio" of which the parameters are derived from numerical integration inside the Earth. These are the next generation of Wahr's model. Presently, we have three transfer functions of this kind. One is that of Dehant and Defraigne (1997), in which the authors incorporate mantle heterogeneities and associated boundary deformations (steady state convection computation) in the initial equilibrium Earth. The second and third are the transfer functions of Schastok (1997) and of Huang et al. (2000) who incorporate second order terms in the boundary conditions and consider a readjustment of the flattening profile with respect to hydrostatic equilibrium, and include directly in the integration, the oceanic contribution for all nutation frequencies, as well as the atmospheric contribution.

(3) The semi-analytical formula of Mathews et al. (1991, 2000a and 2000b) or of Zharov and Pasynok (2000) based also on a fit on VLBI observations. The parameters fitted are physical parameters such as the core and inner core flattenings, the dynamical flattening (for Mathews et al., 1991, 2000a and 2000b, only), the elastic and inelastic parameters (called "compliances" in these works), the coupling constants involved in the electromagnetic coupling at the CMB and the ICB. In the latest version of their model, Mathews et al. (2000a, and 2000b) have incorporated the ocean effects on nutation. This is done by taking into account the frequency resonance to the free core nutation (FCN) of the ocean and the ocean dynamics constrained by Topex/Posseidon data.

As will be stressed also in Level 6 , it is necessary to take into account the effects of the ocean and atmosphere. These can be accounted for by a fit to the VLBI data of a prograde annual nutation (in-phase and out-of-phase parts). The ocean effects are generally computed from tidal maps for particular tides. These maps allow us to correct some of the nutations, but this is not sufficient; it is necessary to correct at other frequencies also. The only models providing that are the model of Mathews et al. (2000a) and the model of Huang et al. (2000).

The comparison between the IERS 96 nutation series and MHB2000 can be done in the time domain by looking at the residuals with respect to the observations corrected also for the FCN mode. This is shown in Figure 1. The same comparison can be done with a nutation series based on numerical integration, as presented in Figure 2. On the other hand, the nutations can be compared in the frequency domain by giving one-by-one their amplitudes with respect to the corresponding adopted IAU 1980 nutation. This is shown in Table 1 for the in-phase component of the four largest nutations and in Figure 3, for the outof-phase components. The advantage of the semi-analytical nutations (or the empirical nutations) over the numerical integration method can immediately be seen from these graphs. In the numerical integration approach, dissipation at 


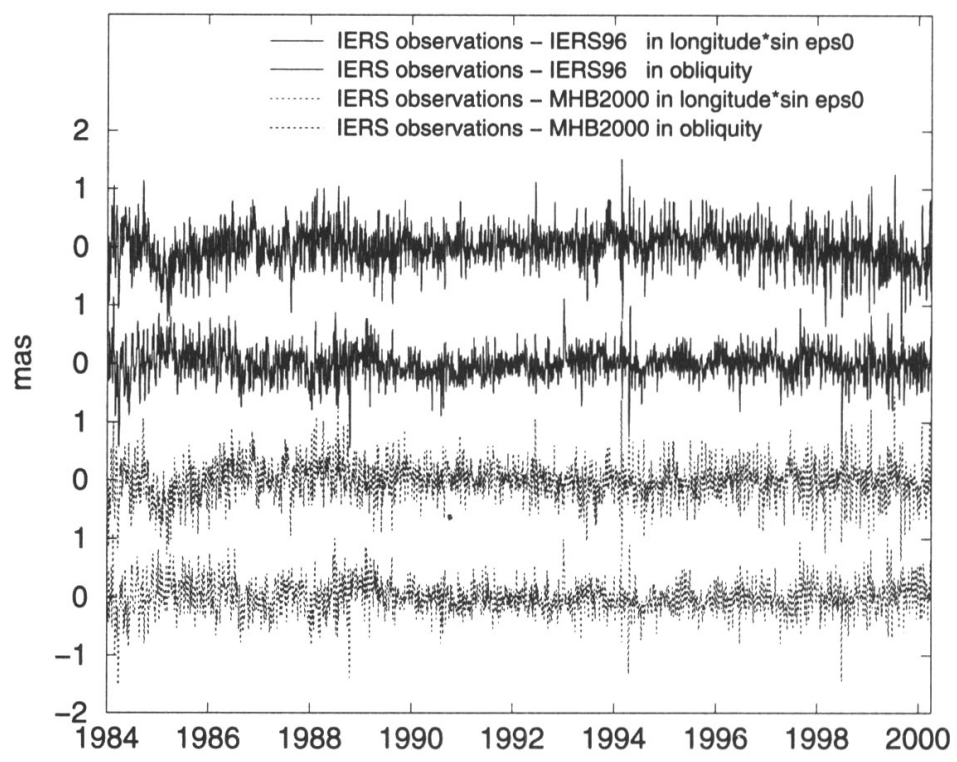

Figure 1. Comparison of the difference between VLBI observation and the IERS96, VLBI observation and the MHB2000.

the CMB is neglected and no FCN dissipation is taken into account. As a result, in particular the large retrograde annual out-of-phase component cannot be explained. The 18.6-year nutation of which the amplitude is quite large suffers from the same problem. The lack of dissipation at the ICB is also partly responsible for that difference.

Although the only model incorporating dissipation and accounting for the ocean at frequencies other than those given by ocean tidal maps is MBH2000 of Mathews et al. (2000a), developments of completely numerical models such as the Dehant and Defraigne (1997) and Huang et al. (2000) should also be encouraged. Empirical formula fit to the observations provide very small residuals as well, but the parameters cannot be interpreted in terms of physics of the Earth's interior.

\section{Level 3: rigid-Earth nutation theories}

The pioneer work of Kinoshita for the adopted IAU 1980 nutation series (Kinoshita, 1977) considered the lunisolar attraction only. Since that time, new nutation series have been derived, which incorporate the direct and indirect planetary effects, the $J_{2}$ tilt effect (related to the effect of the Earth's equatorial bulge on the Moon's orbit and hence on the relative position of the Moon and the Earth), the effects of the nutations on the nutations (related to the changes in the positions of the Earth in space), and the effect of the non-ellipsoidal part of the Earth's gravitational potential. 


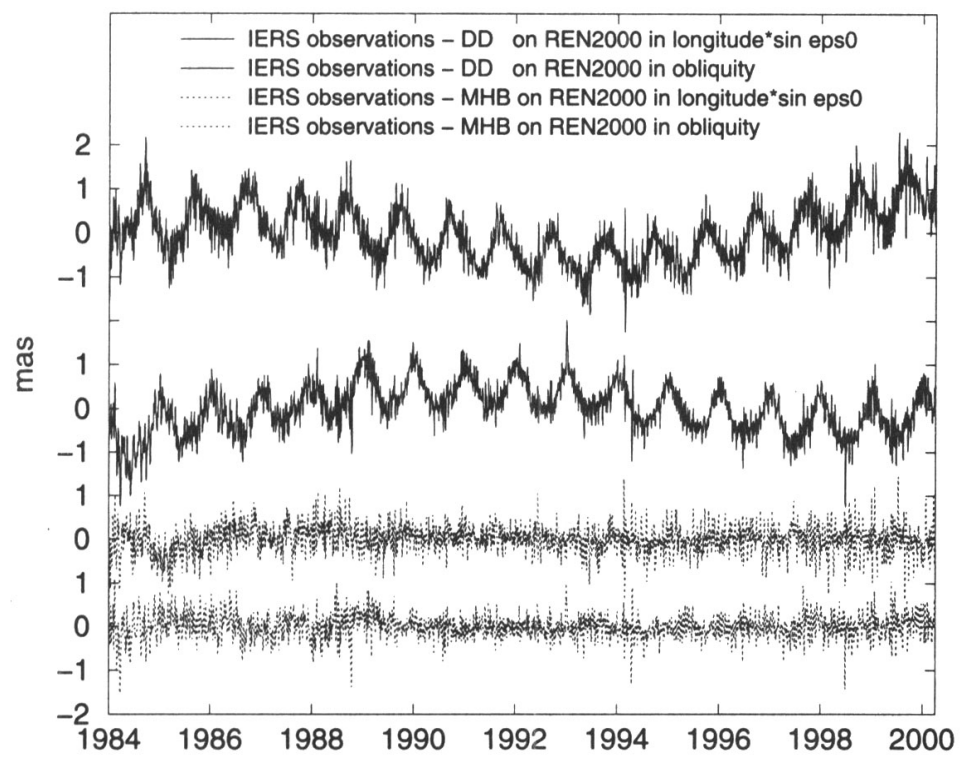

Figure 2. Comparison of the difference between VLBI observation and DD97, VLBI observation and the MHB2000.

Table 1. In-phase components of the four largest residuals with respect to the IAU 1980 nutation series.

$\begin{array}{lcccccccc} & \begin{array}{c}18.6-\mathrm{yr} \\ \text { retro }\end{array} & \begin{array}{c}18.6-\mathrm{yr} \\ \text { pro }\end{array} & \begin{array}{c}1-\mathrm{yr} \\ \text { retro }\end{array} & \begin{array}{c}1-\mathrm{yr} \\ \text { pro }\end{array} & \begin{array}{c}0.5-\mathrm{yr} \\ \text { retro }\end{array} & \begin{array}{c}0.5-\mathrm{yr} \\ \text { pro }\end{array} & \begin{array}{c}13.7-\mathrm{d} \\ \text { retro }\end{array} & \begin{array}{c}13.7-\mathrm{d} \\ \text { pro }\end{array} \\ \text { Mathews et al. 2000 } & -2.71 & 0.03 & -1.99 & -0.01 & -0.04 & 0.59 & 0.02 & -0.12 \\ \text { Ferrándiz \& Getino 2000 } & -2.71 & 0.03 & -1.99 & -0.014 & -0.029 & 0.597 & -0.02 & -0.127 \\ \text { Dehant \& Defraigne 1997 } & -2.37 & -0.09 & -2.02 & -0.04 & -0.03 & 0.66 & 0.01 & -0.17 \\ \text { Schastock 1997 } & -2.65 & 0.01 & -1.98 & 0.05 & -0.03 & 0.66 & 0.01 & -0.13 \\ \text { Huang } \text { et al. 2000 } & -2.76 & -0.1 & -2 & -0.02 & 0.01 & 0.69 & 0.01 & -0.13 \\ \text { Zharov \& Pasynok 2000 } & -3.13 & 0.08 & -2 & -0.01 & 0 & 0.58 & -0.01 & -0.1 \\ \text { Shirai \& Fukushima 2000 } & -2.7 & -0.05 & -2.01 & -0.01 & -0.04 & 0.57 & 0.01 & -0.14 \\ \text { IERS 1996 } & -2.76 & -0.1 & -1.98 & -0.01 & -0.06 & 0.6 & 0.01 & -0.13 \\ \text { Feissel et al. 1998 } & -2.82 & -0.11 & -2.02 & -0.04 & -0.06 & 0.59 & 0.01 & -0.11 \\ \text { Dehant \& Defraigne 1998 } & -2.83 & -0.01 & -2 & -0.01 & -0.04 & 0.59 & 0.02 & -0.1 \\ \text { Herring 2000 } & -2.82 & -0.07 & -1.98 & -0.02 & -0.05 & 0.59 & 0.01 & -0.12\end{array}$



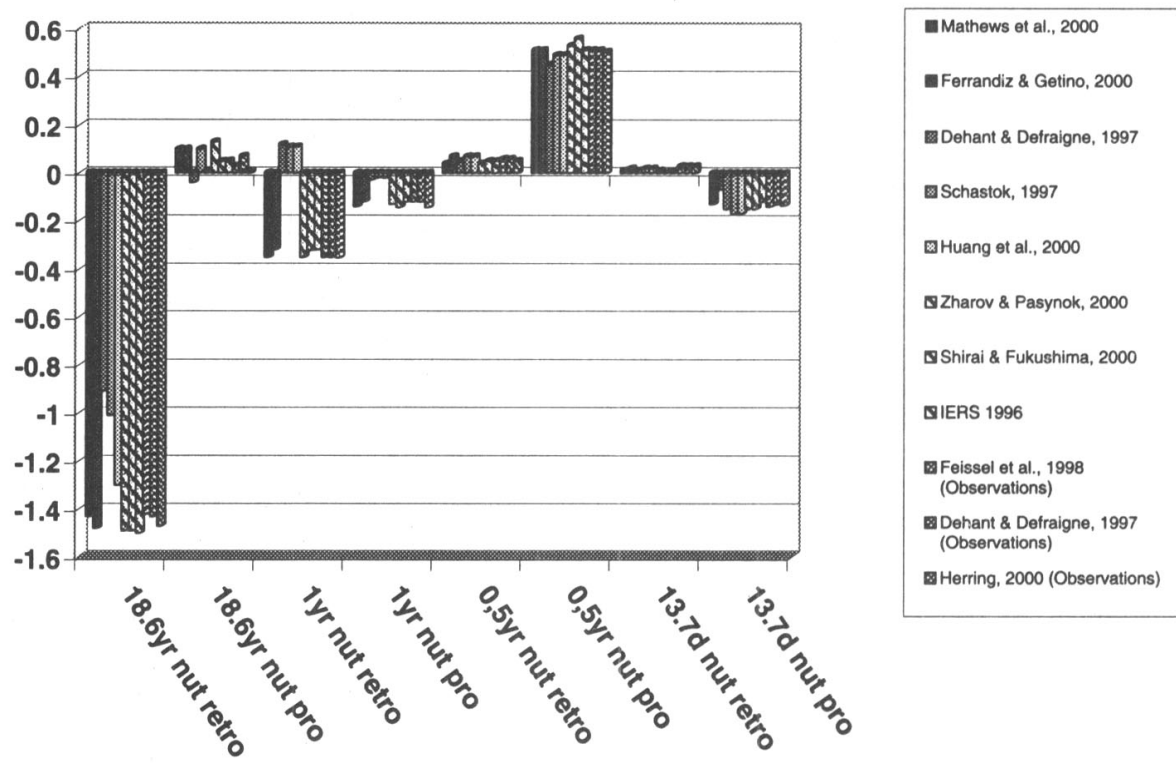

Figure 3. Corresponding out-of-phase components of the four nutations.

There are currently three rigid-Earth nutation series in agreement at a few hundreds of microarcseconds. Two of them have been built using the torque approach: RDAN97 (Roosbeek and Dehant, 1998) and SMART97 (Bretagnon et al., 1998), and one of them, REN2000 (Souchay et al., 1999), has been build using the Hamiltonian approach. The most precise series is SMART97, which is truncated at 0.01 microarcsecond, the other two being truncated at $0.1 \mathrm{mi}-$ croarcsecond. At the 0.1 microarcsecond level, they all have about 1500 terms. It must be noted that the arguments that are used in the harmonic functions for SMART97 are different from those of the two other series. REN2000 and RDAN97 use, for instance, for the most principal nutation, the classical argument $\Omega$ varying with a period of 18.6 years and also containing a secular variation, while the corresponding argument used by SMART 97 does not contain any argument or combination of arguments of which the periods are longer than a few decades; the frequencies in the nutation series will then never be separated by a very small amount corresponding to a very long period. These arguments are certainly more realistic when using a secular variation theory, but they are not widely used in the astronomical community.

Very recently, the mutual influence of UT1 variations and nutations has been studied; this could lead to corrections, namely at the 18.6-year nutation, well above the observational uncertainties. This work has not yet be published but will be very soon (Souchay and Folgueira, 2000; Bretagnon et al., 2000), and should be encouraged. 


\section{Level 4: convolution}

The nutations of a nonrigid Earth are usually obtained from the convolution of a transfer function with a rigid-Earth nutation series. When comparing the various results with the VLBI observations, only the changes in the transfer function are visible. Nevertheless if one wants to deduce the numerical values of the physical parameters from a fit to the VLBI data, it is preferable to use the most precise rigid-Earth nutation theory. The MBH2000 is based on REN2000 for the moment, but the WG has taken some action items for the near future to test the other rigid-Earth nutation series. In Figure 1, the residuals between the observations and MHB2000 and those between the observations and IERS96 are presented. No clear differences can be seen. In Figure 2, the residuals between the observations and MHB2000 and those between the observations and DD97 (Dehant and Defraigne, 1997) are presented. It can immediately be seen that some nutations, those that are influenced by resonance and/or have large amplitudes, are obviously very different.

Besides the method based on convolution between the transfer function and the rigid-Earth nutation series, there exists an approach based on a global or generalized Hamiltonian approach (Ferrándiz and Getino, 2000). In this theory, the authors have computed directly the Hamiltonian for a nonrigid Earth. For the moment, the parameters chosen for fitting the observations are correlated, the ocean corrections are the same as in Dehant and Defraigne (1997) and thus only for a few nutations, and the number of nutation terms is only about 100 . So, while the idea of a general global approach is valuable, the model is not yet competitive with the MBH2000. On the other hand, the parameters of the model are not fitted to VLBI data, but on the empirical model IERS96 itself. Nevertheless, the idea of considering rigid and nonrigid Earth together is really valuable and we have decided to encourage this in the resolution.

Another new interesting method for computing the nutation series for a nonrigid Earth, is the numerical convolution developed by Shirai and Fukushima $(2000 \mathrm{~b})$. These authors have computed the convolution in the time domain instead of using the transfer function in the frequency domain. This has the advantage of reducing the error in the time domain. As stated in their paper, the errors of the current method of the analytical convolution in creating the nutation theory reached about 1 microarcsecond, while those of numerical convolution reached only 1 nano-arcsecond.

\section{Level 5: ocean and atmosphere}

To compute the ocean and atmospheric effects on nutation one can either use the torque approach or the angular momentum approach. The angular momentum approach considers the total angular momentum of the Earth-atmosphere-ocean system to be constant. To any angular momentum change in the superficial fluid layer corresponds an opposite change in the Earth's angular momentum. The knowledge of the time evolution of the fluid angular momentum provides all the information about the time evolution of the Earth's angular momentum and thus about its rotation. The total angular momentum is generally divided into two parts: the matter term, corresponding to a rigid rotation of the fluid layer 
with the Earth, and the motion term, related to the additional relative angular momentum of the fluid layer with respect to the Earth, and related to the winds and currents.

The torque approach is based on the direct computation of the interaction torques between the Earth and the superficial fluids. The three contributions to this torque are the pressure torque, related to the fluid weight acting on the Earth's topography or bathymetry, the gravitational torque, related to the gravitational attraction between the masses inside the Earth and the atmospheric masses, and the friction torque, related to the friction of the winds and currents.

At present, at least one order of magnitude difference exists between the results of the two approaches, the angular momentum approach being closer to the observations. These computations and the understanding of the differences certainly need further investigations. In practice, these computations are not used, instead the annual prograde nutation amplitude and phase are fit to the data.

\section{Level 6: Comparison with observations}

When comparing the well-developed nonrigid-Earth nutation series corrected for the oceanic and atmospheric effects to the VLBI observations (see Figure 4) one immediately sees large residuals for a period close to but greater than one year. It is the effect of the FCN mode excited by the atmosphere, and it can reach a level of 0.2 milliarcseconds from time to time. It is thus necessary to correct for this part that is the equivalent of the Chandler Wobble (CW) in the polar motion. The excitation of this free mode is most probably due to the atmosphere as shown by Gegout et al. (1998). Because of its unpredictible amplitude, the FCN free mode must be observed.

\section{Conclusions}

Working at the level of the input used to compute the transfer functions (seismic model, Level 1), at the level of the transfer functions themselves (Level 2), at the level of the rigid-Earth nutation series (Level 3), at the level of the convolution between the transfer functions and the rigid-Earth nutation series (Level 4), at the level of the corrections for the ocean and atmosphere (Level 5) necessary to compare with the observations (Level 6), we have understood the advantages and inconveniences of the existing models. The models based on numerical integration inside the Earth (which is, in principle, the next generation of the model of the Wahr (1981), the IAU 1980 nutation model) suffer from a lack of dissipation (only mantle inelasticity is taken into account), from the absence of electromagnetic coupling at the liquid core boundaries (CMB and ICB). The semi-analytical models based on a three-layered Earth are able to incorporate that. Empirical formulas fit to VLBI data can provide about the same precision as the semianalytical models, but have to be used with caution in the sense that the results cannot be interpreted in terms of the physics of the Earth's interior. The only model that incorporates the ocean corrections correctly (i.e. at all the nutation frequencies), is the semi-analytical model of Mathews et al. (2000a) called MHB2000. This model corresponds to a semi-analytical formula that must be 


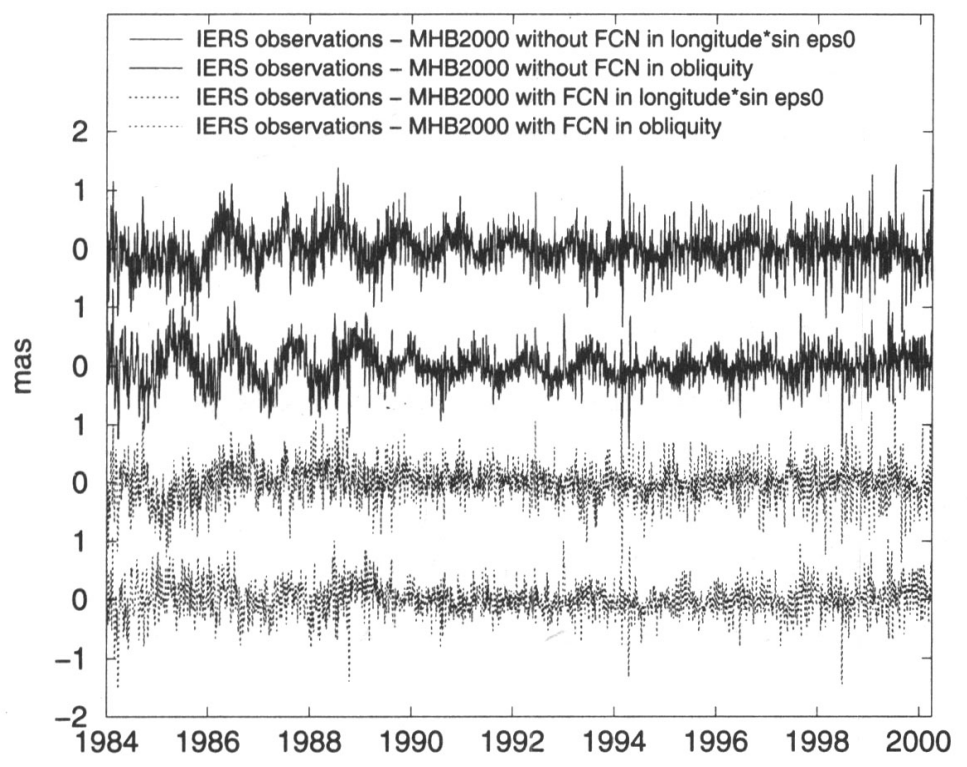

Figure 4. Comparison of the residuals between VLBI observation and MHB2000 without taking out the FCN and with taking it out.

convolved with a rigid-Earth nutation series. The convolution performed on the three different rigid-Earth nutation series (SMART2000, RDAN97, REN2000) can be compared with the observations. The residuals are shown in Figure 5 and are apparently equal for the three series. In the action items of the WG, a comparison with the observations in terms of weighted rms will be performed very soon to determine exactly the best model to be adopted by the IAU. This involves in a first step, a fit to VLBI data (USNO data set) of 21 pairs of nutations and of the precession rate, starting from one of these rigid-Earth nutation series, then in a second step, a new computation of the parameters of MHB2000 nutation model from the three different sets (based on the three different rigidEarth nutation theories). Presently only REN2000 has been used to provide MHB2000. Although the differences could be very small, the WG has decided to test that point before choosing definitely the IAU 2000 model.

As a last remark, we would like to stress the importance of taking into account the mixture between secular variations and nonrigid-Earth nutations and the mutual influences of UT1 variations and the nutations; we are convinced of the appropriateness of such global models in the future. 


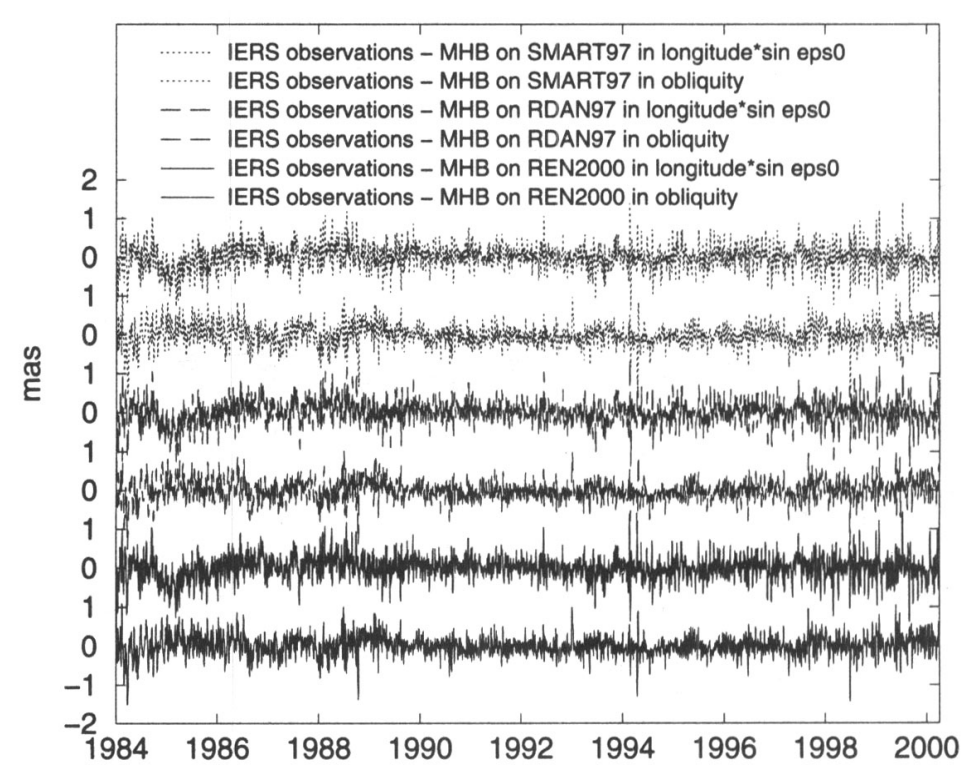

Figure 5. Comparison of the residuals between VLBI observation and MHB2000 convolved with REN2000 (as constructed for), with RDAN97 and with SMART97.

\section{References}

Bretagnon, P., Francou, G., Rocher, P., and Simon, J.-L., 1998, "SMART97 : a new solution for the rotation of the rigid Earth," Astron. Astrophys., 329, 329-338.

Bretagnon, P., Mathews, P.M., Rocher, P., and Simon, J.-L., 2000, "Nonrigid Earth rotation solution," this volume.

Dehant, V., Arias, F., Bizouard, Ch., Bretagnon, P., Brzezi/'nski, A., Buffett, B., Capitaine, N., Defraigne, P., de Viron, O., Feissel, M., Fliegel, H., Forte, A., Gambis, D., Getino, J., Gross, R., Herring, T., Kinoshita, H., Klioner, S., Mathews, P.M., McCarthy, D., Moisson, X., Petrov, S., Ponte, R.M., Roosbeek, F., Salstein, D., Schuh, H., Seidelmann, K., Soffel, M., Souchay, J., Vondrák, J., Wahr, J.M., Weber, R., Williams, J., Yatskiv, Y., Zharov, V. and Zhu, S.Y., 1999, "Considerations concerning the non-rigid Earth nutation theory," Celest. Mech. Dynamical Astron., 72, 245-310.

Dehant, V. and Defraigne, P., 1997, "New transfer functions for nutations of a non-rigid Earth," J. Geophys. Res., 102, 27,659-27,688.

Ferrándiz, J.M. and Getino, J., 2000, "Advances in the unified theory of the rotation of the nonrigid Earth," this volume.

Gegout, P., Hinderer, J., Legros, H., Greff, M. and Dehant, V., 1998, "Influence of atmospheric pressure on the Free Core Nutation, precession and some 
forced nutational motions of the Earth," Phys. Earth planet. Inter., 106, $337-351$.

Herring, T., 2000, personal communication.

Huang, C.L., Jin, W.J., Liao, X.H., 2000, "A new nutation model of non-rigid Earth with ocean and atmosphere," in: McCarthy, D.D. ed., Proc. of IAU Colloquium 180, "Towards Models and Constants for Sub-Microarcsecond Astrometry", March 27-31 2000, Washington DC., USA, this volume.

Kinoshita, H., 1977, "Theory of the rotation of the rigid Earth," Celest. Mech., 15, 277-326.

Kinoshita H., Nakajima K., Kubo Y., Nakagawa I., Sasao T. and Yokohama K., 1979, Note on nutation in ephemerides., Publ. Int. Lat. Obs. Mizusawa, 12, 71-108.

Mathews, P.M., Buffett, B.A., Herring, T.A. and Shapiro, I.I., 1991a, "Forced nutations of the Earth: Influence of inner core Dynamics. I. Theory," $J$. Geophys. Res., 96, 8219-8242.

Mathews, P.M., Buffett, B.A., Herring, T.A. and Shapiro, I.I., 1991b, "Forced nutations of the Earth: Influence of inner core Dynamics. II. Numerical results and comparisons," J. Geophys. Res., 96, 8243-8258.

Mathews, P.M., Herring, T.A., Buffett, B.A., 2000a, "Modeling of nutation-precession: New nutation series for nonrigid Earth, and insights into the Earth's interior," J. Geophys. Res., submitted.

Mathews, P.M., Herring, T.A., Buffett, B.A., 2000b, "Improved Models for Precession and Nutation," this volume.

Roosbeek, F. and Dehant, V., 1998, "RDAN97: an analytical development of rigid Earth nutation series using the torque approach," Celest. Mech. Dynamical Astron., 70, 215-253.

Schastok, J., 1997, "A new nutation series for a more realistic model Earth," Geophys. J. Int., 130, 137-150.

Shirai, T. and Fukushima, T., 2000a, "Improvement of non-rigid Earth nutation theory by adding a model free core nutation term," Astron. J., submitted.

Shirai, T. and Fukushima, T., 2000b, "Numerical convolution in the time domain and its apllication to the non-rigid Earth nutation theory," Astron. J., accepted.

Souchay, J., Loysel, B., Kinoshita, H., and Folgueira, M., 1999, "Corrections and new developments in rigid Earth nutation theory: III. final tables REN2000 including crossed-nutation and spin-orbit coupling effects," Astron. Astrophys., 135, 111-131.

Souchay, J. and Folgueira, M., 2000, "The effect of zonal tides on the dynamical ellipticity of the Earth and its influence on the nutation," Earth, Moon and Planets, in press.

Wahr J.M., 1979, "The tidal motions of a rotating, elliptical, elastic and oceanless Earth," Ph.D. thesis, Univ. of Colo., Boulder, 216 pp.

Wahr, J.M., 1981, "The forced nutations of an elliptical, rotating, elastic and oceanless Earth," Geophys. J. R. astron. Soc., 64, 705-727.

Zharov, V. and Pasynok, S., 2000, personal communication. 